\title{
An empirical method for identifying and translating technical terminology
}

\author{
Sayori Shimohata \\ Rescarch \& Development Group, \\ Oki Electric Industry Co., Ltd. \\ Crystal Tower 1-2-27 Shiromi, \\ Chuo-ku, Osaka 540-6025 Japan \\ shimohata245@oki.co.jp
}

\begin{abstract}
This paper describes a method for retrieving patterns of words and expressions frequently used in a specific domain and building a dictionary for machine translation(MT). The method uses an untagged text corpus in retrieving word sequences and simplified part-of-spcech templates in identifying their syntactic categories. The paper presents experimental results for applying the words and expressions to a patternbased machine translation system.
\end{abstract}

\section{Introduction}

There has been a continuous interest in corpusbased approaches which retrieve words and $\mathrm{ex}-$ pressions in connection with a specific domain (we call them technical terms hereafter). They may correspond to syntactic phrases or components of syntactic relationships and have been found useful in various application areas, including information extraction, text summarization, and machine translation. Among others, a knowledge of technical terminology is indispensable for machine translation because usage and meaning of technical terms are often quite different from their literal interpretation.

One approach for identifying technical terminology is a rule-based approach which learns local syntactic patterns from a training corpus. A variety of methods have been developed within this framework, (Ramshaw, 1995) (Argamon et al., 1999) (Cardic and Pierce, 1999) and achieved good results for the considered task. Surprisingly, though, little work has been devoted to learning local syntactic patterns besides noun phrases. Another drawback of this approach is that it requires substantial training corpora, in many cases with part-of-speech tags.

An alternative approach is a statistical one which retrieves recurrent word sequences as collocations (Smadja, 1993)(Haruno et al., 1996)(Shimoliata et al., 1997). This approach is robust and practical because it uses plain text corpora without any information dependent on a language. Unlike the former approach, this approach extracts various types of local patterns at the same time. Therefore, post-processing, such as part of speech tagging and syntactic category identification, is necessary when we apply them to NLP applications.

This paper presents a method for identifying technical terms from a corpus and applying them to a machine translation system. The proposed method retrieves local patterns by utilizing the n-gram statistics and identifies their syntactic categories with simple part-of-speech templates. We make a machine translation dictionary from the retrieved patterns and translate documents in the same domain as the original corpus.

In the next section, we briefly describe a pattern-based machine translation. The following section explains how the proposed method works in detail. We then present experimental results and conclude with a discussion.

\section{Pattern-based MT system}

A pattern-based MT system uses a set of bilingual patterns(CFG rules) (Abeille et al., 1990) (Takeda, 1996) (Shimohata et al., 1999). In the parsing process, the engine performs a CFGparsing for an input sentence and rewrites trees by applying the source pattcrns. Ierminals and non-terminals are processed under the same framework but lexicalized patterns have priority over symbolized patterns ${ }^{1}$. A plausible parse

\footnotetext{
${ }^{1}$ We define a symbolized pattern as a pattem without a terminal and a lexicalized pattern as that with more than one terminal. we prepares 1000 symbolized patterns and 130,000 lexicalized patterns as a system
} 
tree will be selected among possible parse trees by the number of patterns applied. 'Then the parse tree is transferred into target language by using target patterns which correspond to the source patterns.

Figure 1 shows an example of translation patterns between Fnglish and Japanese. Wach English pattern(a left-half Cl G rule) has corresponding Japanese pattern(a right-hall Cl:G rule). Non-terminals are bracketed with index numbers which represents correspondenco of non-terminals between the source and target pattern.

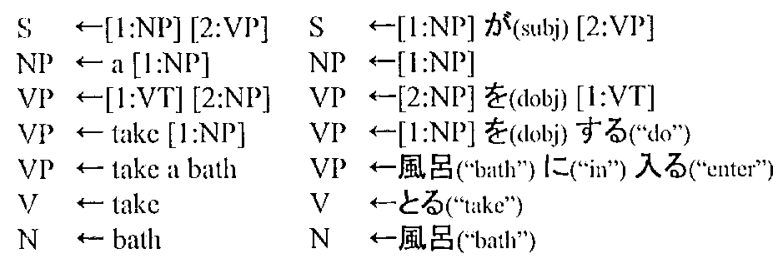

ligure 1: translation patterns

The pattern format is simple but highly descriptive. It can represent complicated linguistic phenomena and even correspondences between the languages with quite diflorent structures. lurthormore, all the knowledge necessary for the translation, whether syntactic or lexical, are compiled in the same pattern format. Owing to these features, we can easily apply the retrieved tochnical torms to a real MI' system.

\section{Algorithm}

ligure 2 shows an outline of the proposed method. The input is an untagged monolingual corpus, while the output is a domain dictionary for machine translation. The process is comprised of 3 phases: retrieving local patterns, assigning their syntactic categories with part-ofspeech(POS) templates, and making translation patterns. 'The dictionary is used when an MT' system translates a text in the same domain as the corpus.

We assume that the input is an English corpus and the dictionary is used for an EnglishJapanese M'I system. In the remainder of this section, we will explain each phase in detail with linglish and Japanese examples.

dictionary.

\subsection{Retrieving local patterns}

We have already proposed a method for retricving word sequences (Shimohata et al., 1997). This method gencrates all n-character (or $n$ word) strings appearing in a text and filters out fragmental strings with the distribution of words adjacent to the strings. This is based on the idea that adjacent words are widely distributed if the string is meaningful, and are localized if the string is a substring of a meaningful string.

The method introduces cntropy value to measture the word distribution. Iet the string be str, the adjacent words $w_{1} \ldots w_{n}$, and the frequency of str freq(str). The probability of each possible adjacent word $p\left(w_{i}\right)$ is then:

$$
p\left(w_{i}\right)=\frac{\operatorname{freq}\left(w_{i}\right)}{\operatorname{freq}(s t r)}
$$

At that time, the entropy of str $H(s t r)$ is defined as:

$$
I I(s t r)=\sum_{i=1}^{n}-p\left(w_{i}\right) \log p\left(w_{i}\right)
$$

Calculating the entropy of both sides of str, the lower one is used as $I(s t r)$. Then the strings whose entropy is larger than a given threshold are retrioved as local patterns.

\subsection{Identifying syntactic categories}

Since the strings are just word sequences, the process gives them syntactic categories. For each str str,

1. assign part-of-specch tags $t_{1}, \ldots t_{n}$ to the component words $w_{1}, \ldots w_{n}$

2. match tag sequence $t_{1}, \ldots t_{n}$ with part-ofspeech templates $I_{i}$

3. give slr corresponding syntactic category $S C_{i}$, if it matches $T_{i}^{\prime}$

\subsubsection{Assigning part-of-speech tags}

'The process uses a simplified part-of-specch set shown in lable 1. Function words are assigned as they are, while content words except for adverb are fallen into only one part of speech word. Four kinds of words "be", "do", "not", and " 10 " are assigned to special tags be, do, not, and to respectively.

There are several reasons to use the simplificd POS tags: 


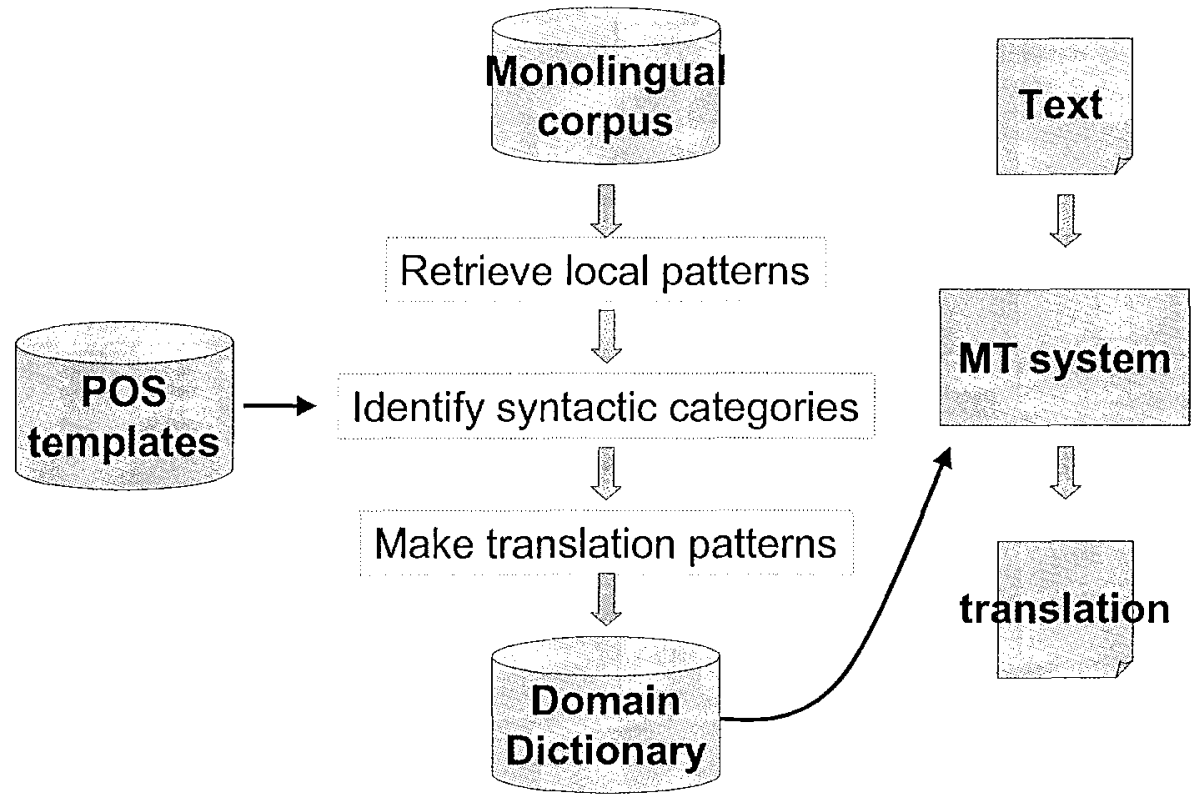

Figure 2: outline

\begin{tabular}{c|c}
\hline l'OS tag & part of speech \\
\hline \hline art & article \\
adv & adverb \\
aux & auxiliary verb \\
conj & conjunction \\
det & detcrminer \\
prep & preposition \\
prn & pronoun \\
punc & punctuation \\
be & "be" \\
do & "do" \\
not & "not" \\
to & "to" \\
word & the others \\
\hline
\end{tabular}

Table 1: part-of-speech tags

- it may sometimos be difficult to identify precise parts of speech in such a local pattern.

- words are often used beyond parts of speech in technical terminology

- it is empirically found that word sequences retrieved through n-gram statistics have distributional concentration on several syn- tactic categories.

Therefore, we think the simplified POS tags are sufficicnt to identify syntactic catcgories.

The word sequence $w_{1}, \ldots w_{n}$ is represented for: a part-of-speech tag secuence $t_{1}, \ldots l_{i}$. Figure 3 shows examples of POS tagging. Italic

$$
\begin{aligned}
& \text { the fuel tank } \\
& \text { art word word } \\
& \text { do this step : } \\
& \text { do det,prn word punc } \\
& \text { to oprn the } \\
& \text { to word art }
\end{aligned}
$$

Figure 3: examples of POS tagging

lines are given word sequences and bold lines are POS tag sequences. If a word falls into two or more parts of speech, all possible POSs will be assigned like "this" in the second example.

\subsubsection{Matching POS templates}

The process identifies a syntactic catcgory(SC) of $s t r$ by checking if str's tag sequence $t_{1}, \ldots$ $t_{n}$ matches a given POS template $T_{i}$. If they 
match, str is given a syntactic category $S C_{i}$ corresponding to $l$. Table 2 shows examples of' POS templates and corresponding SCs ${ }^{2}$.

\begin{tabular}{|c|c|}
\hline $\mathrm{SC}$ & POS tcmplate \\
\hline $\mathrm{N}$ & $(a r l)($ word $\mid \operatorname{conj}) *(w o r d)$ \\
\hline $\mathrm{N}+$ prep & $(a r t)($ word $)+(\operatorname{prcp} \mid t o)(a r t) *$ \\
\hline $\mathrm{V}^{\prime} \mathrm{I}$ & $(a u x \mid$ to $\mid p r n) *($ word $)+(a r l)$ \\
\hline V-ed & $(b c)(w o r d)+(p r c p)(a r t) *$ \\
\hline V & $($ aux $\mid$ to $\mid p r n)($ word $)$ \\
\hline FUNC & $\left(\right.$ art $|a u x| c o n j \mid$ det $\left.\left|p^{r e p}\right| p r n\right)+$ \\
\hline
\end{tabular}

Table 2: POS templates and corresponding SCs

The templates are described in the form of regular expressions(RI) ${ }^{3}$. The first template in table 2, for example, matches a string whose tag sequence begins with an article, contains 0 or more repetitions of content words or conjunctions, and ends with a content word. "the fucl tank" in figure 3 is applied to this templates and given a $\mathrm{SC}$ " $\mathrm{N}$ ".

\subsection{Making translation patterns}

The process converts the strings into translation patterns. The problem here is that we need to generate bilingual translation patterns from monolingual strings. We use heuristic rules on borrowing words from foreign languages ${ }^{4}$.

ligure 4 is an example of conversion rules for generating Fnglish-Japanese translation patterns. To give an example, "to open the" in figure 3 , whose $\mathrm{SC}$ is $\mathrm{VT}$, is converted into the following patterns in accordance with the second rule in figure 4 .

$\mathrm{VP} \leftarrow$ open $[1: \mathrm{NP}]$

$\mathrm{VP} \leftarrow[1: \mathrm{NP}] を(\mathrm{dobj})$ open する(“do”)

\footnotetext{
${ }^{2}$ Note that the P'OS templates are strongly dependent on the features of $n$-gram strings.

3 "*" canses the resulting RE to match 0 or more repetitions of the preceding Rli. " + " causes the resulting RE to match 1 or more repetitions of the preceding Rli. "" creates a RE expression that will match either right or left of "l". "(...)" indicates the start and end of a group.

${ }^{4}$ In Japanese, forcign words, especially in technical terminology, are often used as they are in hatakana (the phonetic spelling for foreign words) followed by function words which indicate their parts of specch l'or example, English verbs are followed by "sure", a verb which means " $d o "$ in linglish.
}

If $\mathrm{SC}$ is $\mathrm{N}$, delete art and generate:
$\mathrm{NP} \leftarrow s t r$

$\mathrm{NP}^{2} \leftarrow s t r$

If SC is VT, delete (aux|to|prn) and art and generate:

$\mathrm{VP} \leftarrow \operatorname{str}[1: \mathrm{NP}]$

$\mathrm{VP} \leftarrow[1: \mathrm{NP}]$ を(dobj) str する("do")

If SC is V, delete (aux|to|prn) gencrate:

$$
\begin{aligned}
& V \leftarrow s t r \\
& V \leftarrow s t r \text { する(“do") }
\end{aligned}
$$

Figure 4: conversion rules for generating translation patterns

\section{Evaluation}

Wo have tested our algorithm in building a domain dictionary and making a translation with it. A corpus used in the experiment is a computer manual comprising 167,023 words (in 22,041 sentences).

The corpus contains 24,137 n-grams which appear more than twice. Among them, 7,616 strings are extracted over the entropy threshold 1. Table 3 is a list of top 20 strings (except for single words and function word sequences) retrieved from the test corpus.

These strings are categorized into 1,239 POS patterns. lable 4 is a list of top 10 POS patterns and the numbers of strings classified into them. In this experiment, the top 10 POS patterns account for $49.4 \%$ of all P'OS patterns. It substantiates the fact that the retrieved strings tend to concentrate in certain POS patterns.

\begin{tabular}{r|l}
\hline freq & \multicolumn{1}{|c}{ POS } \\
\hline \hline 1886 & word \\
553 & word word \\
368 & art word \\
229 & art word word \\
160 & word prep \\
158 & word art \\
121 & word word word \\
108 & to word \\
101 & prep art word \\
81 & prep word \\
\hline
\end{tabular}

Table 4: top 10 POS patterns 


\begin{tabular}{c|c|l||c|c|l}
\hline$H($ str $)$ & freq(str) & \multicolumn{1}{c|}{ str } & $H($ str $)$ & freq(str) & \multicolumn{1}{c}{ str } \\
\hline \hline 5.51 & 247 & see also & 3.55 & 552 & the client \\
4.48 & 1499 & the server & 3.54 & 209 & use the \\
4.46 & 100 & click OK & 3.46 & 209 & the user \\
3.92 & 106 & use this function & 3.46 & 168 & click the \\
3.79 & 163 & the function & 3.44 & 172 & the catalog agent \\
3.76 & 309 & the following & 3.36 & 192 & the request \\
3.67 & 297 & the file & 3.29 & 132 & on page \\
3.58 & 36 & in the Server Manager & 3.23 & 213 & a specified \\
3.56 & 169 & using the & 3.22 & 71 & if you want to \\
3.55 & 180 & CGI programs & 3.22 & 575 & your server \\
\hline
\end{tabular}

Table 3: top 20 strings

In the matching process, we prepared 15 templates and $6 \mathrm{SCs}$. Table 5 is a result of SC identification. 2,462 strings(32.3\%) are not matched to any templates. The table indicates that most strings retrieved in this method are identified as $\mathrm{N}$ and NP. It is quite reasonable because the majority of the technical terms are supposed to be nouns and noun phrases.

\begin{tabular}{c|r}
\hline SC & number of patterns \\
\hline NP & 722 \\
N+prep & 200 \\
VP & 32 \\
VP+prep & 10 \\
VT & 177 \\
V & 78 \\
\hline
\end{tabular}

Table 5: result of SC identification

The retrieved translation patterns total 1,219. Figure 5 shows an example of translation patterns retrieved by our method.

We, then, converted them to an MT dictionary and made a translation with and without it. Table 6 summarizes the evaluation results translating randomly selected 1,000 sentences from the test corpus. Compared with the translations without the dictionary, the translations with the dictionary improved 571 in parsing and word selection.

Figure 6 illustrates changes in translations. Each column consists of an input sentence, a translation without the dictionary, and a translation with the dictionary. Bold English words

\begin{tabular}{lr}
\hline improved in parsing & 104 \\
improved in word selection & 467 \\
about the same & 160 \\
same & 212 \\
not improved & 57 \\
\hline total & 1000 \\
\hline
\end{tabular}

Table 6: Translation evaluation results

correspond to underlined Japanese.

First two examples show improvement in word selection. The translations of "map(verb)" and "exec" are clianged from word-for-word translations to non-translation word sequences. Although "to make a map" and "exective" are not wrong translations, they are irrelevant in the computer manual context. On the contrary, the domain dictionary reduces confusion caused by the wrong word selection.

Wrong parsing and incomplete parsing are also reduced as shown in the next two examples. In the third example, "Next" should be a noun, while it is usually used as an adverb. The domain dictionary solved the syntactic ambiguity properly because it has exclusive priority over system dictionaries. In the forth example, "double-click" is an unknown word which could cause incomplete parsing. But the phrase was parsed as a verb correctly.

The last one is an wrong example of Japanese verb selection. That was a main cause of errors and declines. The reason why the undesirable Japanese verbs were selected is that 


$$
\begin{array}{ll}
N P \leftarrow \text { fully-qualified domain name } & N P \leftarrow \text { fully-qualified domain name } \\
N P \leftarrow \text { text search engine } & N P \leftarrow \text { text search engine } \\
N P \leftarrow \text { access log for }[1: N P] & N P \leftarrow[1: N P] \text { ("of") access log } \\
V P \leftarrow \text { save [1:NP] } & V P \leftarrow[1: N P \text { を(dobj) save する("do") } \\
V \leftarrow \text { deallocate } & V \leftarrow \text { deallocatcする("do") }
\end{array}
$$

l'igure 5: the retrieved translation patterms

Type the URL prefix you want to map.

あなたが地図("map")を(dobj)作り("make") たいURL 接頭辞をタイプしなさい。 あなたが吕 し(“perform mapping”)たいURL prefix をタイプしなさい。

The exec tag allows an ITTML file to execute an arbitrary program on the server; 幹部タグ(“cxective's tag”) はサーバーで HTML ファイルが任意なプログラムを 実行するのを許す；

exectag は HTML ファイルが server の任意なプログラムを実行するのを許す；

Type the full name of your server, and then click Next.

次に("then") あなたのサーバーの完全な名前をタイプしてクリックしなさい。

あなたの server の完全な名前をタイプして Next を clickしなさい。

\author{
Go to the Control Pancl and double-click the Services icon. \\ Control Pancl へ行きなさい、そうすれば二重な・("double-") は Services アイコンを \\ クリックする("click")。 \\ Control Pancl へ行って Services icon をdouble-click し("double-click")なさい。 \\ Setting additional document directories \\ 追加のドキュメントディレタトリを置く("put,place”)こと \\ 追加の documentに directory 老課する("assign, imposc")こと
}

Figure 6: example sentences in the test corpus

the method added default semantic information to the retrieved nouns and noun phrases. Wo hope to overcome it by a model that classifies noun phrases, for example using verb-noun or adjective-noun relations.

\section{Related work}

As mentioned in section 1, there are two approaches in corpus-based technical term retricval: a rulc-based approach and a statistical approach. Major differences between the two arc:

- the former uses a tagged corpus while the latter uses an untagged one.
- the former retrieves words and phrases with a designated syntactic category while the latier retrieves that with various syntactic categories at the same time.

Our method uses the latter approach because we think it more practical both in resources and in applications.

For comparison, we refer here to Smadja's method (1993) because this method and the proposed method have much in common. In both cases, technical terms are retrieved from an untagged corpus with $n$-gram statistics and given syntactic categories for NJP' applications. The methods are different in that Smadja uses a 
parser for syntactic category identification while we use POS templates. A parser may add more precise syntactic category than POS templates. However, we consider it not to be critical under the specific condition that the variety of input patterns is very small. In terms of portability, the proposed method has an advantage. Actually, adding POS templates is not so time consuming as developing a parser.

We have applied the translation patterns retrieved by this method to a real MT system. As a result, $57.1 \%$ of translations were improved with 1,219 translation patterns. To our knowledge, little work has gone into quantifying its effectiveness to NLP applications. We recognize that the method leaves room for improvement in making translation patterns. We, therefore, plan to introduce techniques for finding translational equivalent from bilingual corpora (Melamed, 1998) to our method.

\section{Conclusion}

We have presented a method for identifying technical terminology and building a domain dictionary for MT. Applying the method to a technical manual in English yielded positive results. We have found that the proposed method would dramatically improve the performance of translation. In the future work, wo plan to investigate the availability of POS patterns which are not categorized into any SCs.

\section{References}

Abeille A., Schabes Y., and Joshi A. K. 1990. "Using Lexicalized Tags for Machine Translation". In Proceedings of the International Conference on Computational Linguistics(COLING), pages 1-6.

Argamon, S., Dagan, I., and Krymolowski, Yuval. 1999. A Memory-Based Approach to Learning Shallow Natural Language Patterns. In Proceedings of the 17th COLING and the 36th Annual Meeting of ACL, pages $67-73$.

Cardie, C. and Pierce, D. 1999. The Role of Lexicalization and Pruning for Base Noun Phrase Grammars In Proceedings of the 16th National Conference on Artificial Intelligence, pages 423-430.

Haruno, M., Ikehara, S., and Yamazaki, T. 1996. Learning Bilingual Collocations by
Word-Level Sorting. In Procedings of the 16th COLING, pages 525-530.

Melamed,I.D. 1998. Empirical Methods for MT' Lexicon Development In Gerber, L. and Farwoll, D. Eds. Machine Translation and the Information Soup, Springer-Verlag.

Ramshaw, L.A., and Marcus, M.P. 1995. Text Chunking using Transformation-Based Jearning In Proceedings of the 3rd Workshop on Very Large Corpora, pages 82-94.

Shimohata,S., Sugio,T., and Nagata,J. 1997. Retrieving Collocations by Co-occurrences and Word Order Constraints. In Proceedings of the 35th Annual Meeting of ACL, pages 476-481.

Shimohata, S. et al. 1999. "Machine Translation System PENSE]: System Design and Implementation," In Proceedings of Machine Translation Summit VII, pp.380-384.

Smadja,F.A. 1993. Retrieving Collocations from Text: Xtract. In Computational Linguistics, 19(1), pages 143-177.

Takeda K. 1996. "Pattern-Based Context-Tree Grammars for Machine Translation". In Proceedings of the 34th Annual Meeting of ACL, pages $144-151$. 